\title{
A Novel Peridynamic Mindlin Plate Formulation Without Limitation on Material Constants
}

\author{
Zhenghao Yang $^{1} \cdot$ Erkan Oterkus $^{1}$ (D) . Selda Oterkus ${ }^{1}$
}

Received: 27 May 2020 / Accepted: 22 January 2021 / Published online: 24 March 2021

(c) The Author(s) 2021

\begin{abstract}
In this study, a new peridynamic Mindlin plate formulation is introduced by utilising EulerLagrange equations. The classical strain energy density of a material point is converted to its corresponding peridynamic form by using Taylor's expansions. The formulation is suitable for thick plates by considering the transverse shear deformation. Material constants do not have any limitation in the current formulation. Different types of boundary conditions are considered in numerical examples including simply supported, clamped and mixed (clamped-supported). To verify the current formulation, peridynamic solutions of the transverse displacements and rotations are compared against solutions obtained from finite element analysis.
\end{abstract}

Keywords Mindlin plate $\cdot$ Peridynamics $\cdot$ Non-local $\cdot$ Transverse shear deformation

\section{Introduction}

Solid mechanics is an important research area focusing on how the materials and structures deform if they are subjected to external loading conditions. As a result of the deformation, permanent damages can occur including plasticity and crack occurrence. Predicting crack initiation and propagation is still one of the challenging areas of solid mechanics. Moreover, with the advancement of technology, interest on nano-scale structures is increasing. Analysis of nano-scale structures can require non-classical approaches including length scale parameters. There are various non-classical approaches available in the literature, and amongst these, peridynamics [1] is the focus of this study. There has been a rapid progress in peridynamics research during the recent years [2-24]. An extensive review of peridynamics is given by Javili et al. [25].

Original peridynamic formulation is suitable for analysis of three-dimensional structures. However, this formulation can be computational expensive for structures which have special shapes that can be considered as beams, plates and shells. To model such structures, standard formulation should be modified by taking into account rotational degrees

Erkan Oterkus

erkan.oterkus@strath.ac.uk

1 PeriDynamics Research Centre, Department of Naval Architecture, Ocean and Marine Engineering, University of Strathclyde, 100 Montrose Street, Glasgow G4 0LZ, UK 
of freedom. Hence, three-dimensional geometries can be represented by one-dimensional models as beams or two-dimensional models as plates and shells. Such formulations are currently available for peridynamics. For instance, a peridynamic formulation to analyse thin plates was introduced in [26]. A non-ordinary state-based Euler-Bernoulli beam formulation was developed in [27]. In another study [28], they extended this formulation to represent the Kirchhoff-Love plate theory. State-based peridynamics was used to develop peridynamic Euler beam and Kirchhoff plate formulations in [29] and [30], respectively. To analyse thick plates, it is essential to take into account transverse shear deformations. Transverse shear deformations were taken into account in [31] as a characteristic of peridynamic Mindlin plate formulation. However, this formulation is limited to Poisson's ratio of $1 / 3$ for isotropic materials.

Hence, a new peridynamic Mindlin plate formulation is introduced in this study which does not have any limitations on material constants. The formulation is developed by using the Euler-Lagrange equation in conjunction with Taylor's expansion. To verify the formulation, several numerical examples are considered for a Mindlin plate under different boundary conditions, and peridynamic and finite element analysis results are compared with each other.

\section{Classical Mindlin Plate Formulation}

Mindlin developed a plate theory for thick plates by considering transverse shear deformation. According to Mindlin plate theory, a transverse normal to the mid-plane of the plate in the undeformed state remains straight, and there is no change in its length during deformation. The displacement components of any material point can be represented in terms of mid-plane ( $x y$ plane) displacements and rotations as

$$
\begin{gathered}
u(x, y, z, t)=z \theta_{x}(x, y, t) \\
v(x, y, z, t)=z \theta_{y}(x, y, t) \\
w(x, y, z, t)=w(x, y, t)
\end{gathered}
$$

where $\theta_{x}$ and $\theta_{y}$ denote the mid-plane rotations about positive $y$-direction and negative $x$-direction, respectively. Moreover, $w$ denotes the mid-plane transverse displacements. The positive set of the degrees-of-freedom is shown in Fig. 1.

Thus, the strain-displacement relationships can be written as

$$
\begin{gathered}
\varepsilon_{x x}=z \frac{\partial \theta_{x}}{\partial x} \\
\varepsilon_{y y}=z \frac{\partial \theta_{y}}{\partial y} \\
\varepsilon_{x y}=\varepsilon_{y x}=\frac{1}{2} z\left(\frac{\partial \theta_{x}}{\partial y}+\frac{\partial \theta_{y}}{\partial x}\right)
\end{gathered}
$$




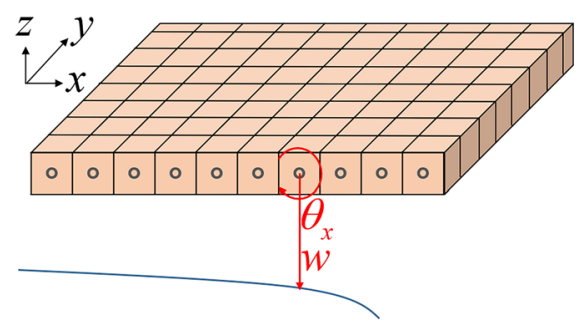

(a)

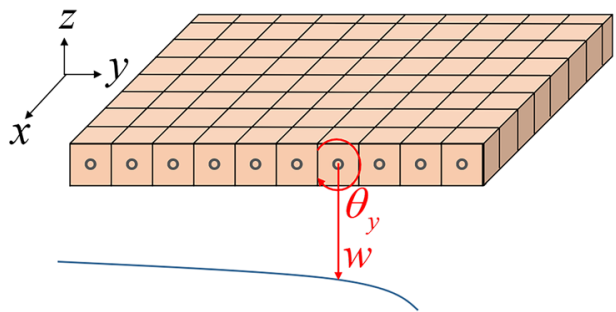

(b)

Fig. 1 Degrees-of-freedom in peridynamic Mindlin formulation. a $x z$-plane. $\mathbf{b} y z$-plane

$$
\begin{gathered}
\gamma_{x z}=\kappa_{s}\left(\theta_{x}+\frac{\partial w}{\partial x}\right) \\
\gamma_{y z}=\kappa_{s}\left(\theta_{y}+\frac{\partial w}{\partial y}\right) \\
\varepsilon_{z z}=0
\end{gathered}
$$

where $\kappa_{s}$ is introduced as shear coefficient. Equations (4-11) can be also expressed in indicial notation as

$$
\begin{gathered}
\varepsilon_{I J}=\frac{1}{2} z\left(\frac{\partial \theta_{I}}{\partial x_{J}}+\frac{\partial \theta_{J}}{\partial x_{I}}\right) \\
\gamma_{I 3}=\kappa_{s}\left(\theta_{I}+\frac{\partial w}{\partial x_{I}}\right)
\end{gathered}
$$

Note that the subscript indices, $I, J, \cdots=1(=x), 2(=y)$, and this convention will be applied throughout this study.

The stress-strain relationships can be written for isotropic materials as:

$$
\begin{gathered}
\sigma_{x x}=\frac{E}{1-v^{2}}\left(\varepsilon_{x x}+v \varepsilon_{y y}\right) \\
\sigma_{y y}=\frac{E}{1-v^{2}}\left(\varepsilon_{y y}+v \varepsilon_{x x}\right) \\
\sigma_{x y}=\frac{E}{2(1+v)}\left(\varepsilon_{x y}+\varepsilon_{y x}\right) \\
\tau_{x z}=G \gamma_{x z} \\
\tau_{y z}=G \gamma_{y z}
\end{gathered}
$$


where $E, G$, and $\nu$ represent elastic modulus, shear modulus and Poisson's ratio, respectively. Note that the transverse normal stress, $\sigma_{z z}$, is considered to be small compared with in-plane stresses. Thus, it is discarded from the stress components set for simplification. The stresses can also be written in indicial notation as:

$$
\begin{gathered}
\sigma_{I J}=C_{I J K L} \varepsilon_{K L} \\
\tau_{I 3}=G \gamma_{I 3}
\end{gathered}
$$

where

$$
C_{I J K L}=\frac{E}{1-v^{2}}\left[\frac{1-v}{2}\left(\delta_{I L} \delta_{J K}+\delta_{I K} \delta_{J L}\right)+v \delta_{I J} \delta_{K L}\right]
$$

The linear elastic strain energy density of the Mindlin plate can be expressed as

$$
\tilde{W}=\frac{1}{2}\left(\sigma_{I J} \varepsilon_{I J}+\tau_{I 3} \gamma_{I 3}\right)
$$

Inserting Eqs. (10), (11) and (17) into Eq. (19) and rearranging indices yields

$$
\begin{aligned}
\tilde{W}= & \frac{E}{1-v^{2}} z^{2}\left[\frac{1-v}{4}\left(\frac{\partial \theta_{I}}{\partial x_{J}} \frac{\partial \theta_{I}}{\partial x_{J}}+\frac{\partial \theta_{I}}{\partial x_{J}} \frac{\partial \theta_{J}}{\partial x_{I}}+\frac{\partial \theta_{I}}{\partial x_{I}} \frac{\partial \theta_{J}}{\partial x_{J}}\right)+\frac{3 v-1}{4} \frac{\partial \theta_{I}}{\partial x_{I}} \frac{\partial \theta_{J}}{\partial x_{J}}\right] \\
& +\kappa_{s}^{2} \frac{G}{2}\left(\theta_{I}+\frac{\partial w}{\partial x_{I}}\right)\left(\theta_{I}+\frac{\partial w}{\partial x_{I}}\right)
\end{aligned}
$$

For a particular material point on the mid-plane, the average strain energy density can be reasonably calculated by integrating the strain energy density function, Eq. (21), through the transverse direction and dividing by the thickness as

$$
\begin{aligned}
W= & \frac{E}{1-v^{2}} \frac{h^{2}}{12}\left[\frac{1-v}{4}\left(\frac{\partial \theta_{I}}{\partial x_{J}} \frac{\partial \theta_{I}}{\partial x_{J}}+\frac{\partial \theta_{I}}{\partial x_{J}} \frac{\partial \theta_{J}}{\partial x_{I}}+\frac{\partial \theta_{I}}{\partial x_{I}} \frac{\partial \theta_{J}}{\partial x_{J}}\right)+\frac{3 v-1}{4} \frac{\partial \theta_{I}}{\partial x_{I}} \frac{\partial \theta_{J}}{\partial x_{J}}\right] \\
& +\kappa_{s}^{2} \frac{G}{2}\left(\theta_{I}+\frac{\partial w}{\partial x_{I}}\right)\left(\theta_{I}+\frac{\partial w}{\partial x_{I}}\right)
\end{aligned}
$$

\section{Peridynamic Mindlin Plate Formulation}

Peridynamics (PD) is a continuum mechanics formulation in which material points can interact with each other in a non-local manner. The range of non-local interactions is defined as "horizon," $H$. The equation of motion peridynamics can be expressed in discrete form as

$$
\rho_{(k)} \ddot{\mathbf{u}}_{(k)}=\sum_{j=1}^{N} \mathbf{f}_{(k)(j)} V_{(j)}+\mathbf{b}_{(k)}
$$

for the material point $k$ where $N$ is the number of material points inside the horizon, $\rho$ is density, $\mathbf{u}$ is the displacement, $t$ is time, $V$ is volume and $\mathbf{b}$ is the body load vector. The interaction force vector, $\mathbf{f}_{(k)(j)}$, between material points $k$ and $j$ can be defined as (see Fig. 2) 


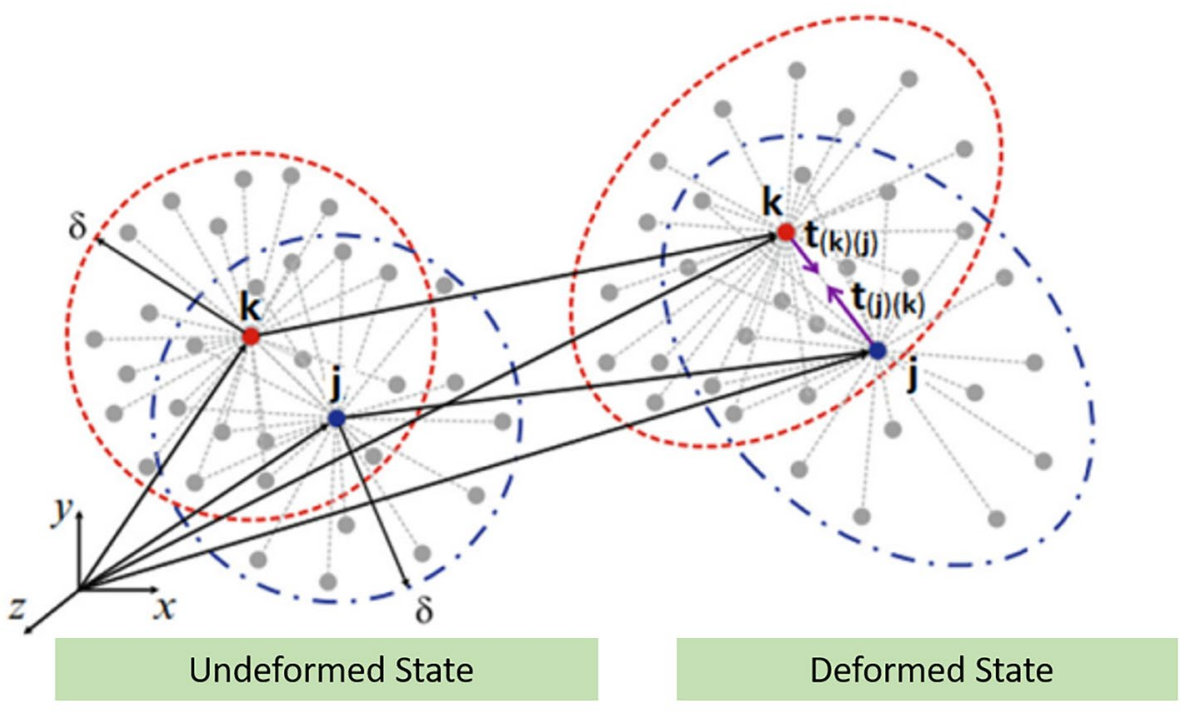

Fig. 2 PD force density factor and horizon [32]

$$
\mathbf{f}_{(k)(j)}=\mathbf{t}_{(k)(j)}-\mathbf{t}_{(j)(k)}
$$

The PD equations of motion can be obtained from Euler-Lagrange's equation as:

$$
\frac{d}{d t} \frac{\partial L}{\partial \dot{\mathbf{u}}_{(k)}}-\frac{\partial L}{\partial \mathbf{u}_{(k)}}=0
$$

where $L$ is the Lagrangian which is the difference between the total kinetic energy, $T$, and total potential energy, $U$, that can be expressed as

$$
T=\frac{1}{2} \int_{A} \int_{-h / 2}^{h / 2} \rho \dot{\mathbf{u}}(x, z, t) \cdot \mathbf{u}(x, z, t) d z d A
$$

and

$$
U=\sum_{k} W_{(k)} V_{(k)}-\sum_{k} \mathbf{b}_{(k)} \cdot \mathbf{u}_{(k)} V_{(k)}
$$

where the generalised displacement vector, $\mathbf{u}$, and generalised body force density vector, $\mathbf{b}$, can be defined as

$$
\mathbf{u}=\left(\begin{array}{lll}
\theta_{1} & \theta_{2} & w
\end{array}\right)^{T}
$$

and

$$
\mathbf{b}=\left(\begin{array}{lll}
b_{\theta_{1}} & b_{\theta_{2}} & b_{z}
\end{array}\right)^{T}
$$

Here, $b_{\theta}$ and $b_{z}$ correspond to moment and transverse force, respectively. Inserting Eqs. (1), (2) and (3) into (26) and discretizing the function gives the kinetic energy of the system as 


$$
T=\frac{1}{2} \sum_{k} \rho_{(k)}\left(\frac{h^{2}}{12}\left(\dot{\theta}_{1}^{(k)}\right)^{2}+\frac{h^{2}}{12}\left(\dot{\theta}_{2}^{(k)}\right)^{2}+\dot{w}_{(k)}^{2}\right) V_{(k)}
$$

Assuming the system is holonomic, the first term of Euler-Lagrange equation can be obtained by substituting Eq. (30) into (25):

$$
\frac{d}{d t} \frac{\partial L}{\partial \dot{\mathbf{u}}_{(k)}}=\frac{d}{d t} \frac{\partial T}{\partial \dot{\mathbf{u}}_{(k)}}=\rho_{(k)}\left\{\begin{array}{c}
\frac{h^{2}}{12} \ddot{\theta}_{1}^{(k)} \\
\frac{h^{2}}{12} \ddot{\theta}_{1}^{(k)} \\
\ddot{w}_{(k)}
\end{array}\right\} V_{(k)}
$$

In PD theory, the strain energy density has a non-local form. Therefore, the strain energy of a certain material point $k$ can be expressed as

$$
W_{(k)}=W_{(k)}\left(\mathbf{u}_{(k)}, \mathbf{u}_{\left(1^{k}\right)}, \mathbf{u}_{\left(2^{k}\right)}, \mathbf{u}_{\left(3^{k}\right)}, \cdots\right)
$$

where $\mathbf{u}_{(k)}$ represents the displacement vector of the material point $k$, and $\mathbf{u}_{\left(i^{k}\right)}(i=1,2,3, \cdots)$ represents the displacement vector of the $i$ th material point inside the horizon of the material point $k$.

The total potential energy given in Eq. (27) can then be written as

$$
U=\sum_{k} W_{(k)}\left(\mathbf{u}_{(k)}, \mathbf{u}_{\left(1^{k}\right)}, \mathbf{u}_{\left(2^{k}\right)}, \mathbf{u}_{\left(3^{k}\right)}, \cdots\right) V_{(k)}-\sum_{k} \mathbf{b}_{(k)} \cdot \mathbf{u}_{(k)} V_{(k)}
$$

Thus, the second term in Eq. (25) can be evaluated as

$$
\begin{aligned}
-\frac{\partial L}{\partial \mathbf{u}_{(k)}} & =\frac{\partial}{\partial \mathbf{u}_{(k)}} \sum_{n} W_{(n)}\left(\mathbf{u}_{(n)}, \mathbf{u}_{\left(1^{n}\right)}, \mathbf{u}_{\left(2^{n}\right)}, \mathbf{u}_{\left(3^{n}\right)}, \cdots\right) V_{(n)}-\frac{\partial}{\partial \mathbf{u}_{(k)}} \sum_{n} \mathbf{b}_{(n)} \cdot \mathbf{u}_{(n)} V_{(n)} \\
& =\sum_{n} \frac{\partial W_{(n)}}{\partial \mathbf{u}_{(k)}}\left(\delta_{n k}+\delta_{n i^{k}}\right) V_{(n)}-\frac{\partial}{\partial \mathbf{u}_{(k)}} \sum_{n} \mathbf{b}_{(n)} \delta_{n k} V_{(n)}=\left(\frac{\partial W_{(k)}}{\partial \mathbf{u}_{(k)}} V_{(k)}+\sum_{j} \frac{\partial W_{(j)}}{\partial \mathbf{u}_{(k)}} V_{(j)}\right)-\mathbf{b}_{(k)} V_{(k)}
\end{aligned}
$$

Inserting Eqs. (31) and (34) into Euler-Lagrange equation yields:

$$
\rho_{(k)}\left\{\begin{array}{c}
\frac{h^{2}}{12} \ddot{\theta}_{1}^{(k)} \\
\frac{h^{2}}{12} \ddot{\theta}_{1}^{(k)} \\
\ddot{w}_{(k)}
\end{array}\right\} V_{(k)}=-\left\{\begin{array}{l}
\frac{\partial W_{(k)}}{\partial \theta_{1}^{(k)}} V_{(k)}+\sum_{j} \frac{\partial W_{(j)}}{\partial \theta_{1}^{(k)}} V_{(j)} \\
\frac{\partial W_{(k)}}{\partial \theta_{2}^{(k)}} V_{(k)}+\sum_{j} \frac{\partial W_{(j)}}{\partial \theta_{2}^{(k)}} V_{(j)} \\
\frac{\partial W_{(k)}}{\partial w_{(k)}} V_{(k)}+\sum_{j} \frac{\partial W_{(j)}}{\partial w_{(k)}} V_{(j)}
\end{array}\right\}+\left\{\begin{array}{l}
b_{\theta_{1}}^{(k)} \\
b_{\theta_{1}}^{(k)} \\
b_{z}^{(k)}
\end{array}\right\} V_{(k)}
$$

As explained in Appendix, the strain energy densities of the material point $k$ and $j$ can be expressed in peridynamic form by transforming all differential terms in Eq. (22) as

$$
\begin{aligned}
& W_{(k)}=\frac{E}{1-v^{2}} \frac{h^{2}}{12}\left[\begin{array}{l}
\frac{1-v}{4}\left(\frac{12}{\pi \delta^{2} h} \sum_{i} \frac{\left(\theta_{I}^{\left(k^{k}\right)}-\theta_{I}^{(k)}\right)\left(\theta_{J}^{\left(i^{k}\right)}-\theta_{J}^{(k)}\right)}{\xi_{\left(i^{k}\right)(k)}^{(k)}} n_{I}^{\left(i^{k}\right)(k)} n_{J}^{\left(i^{k}\right)(k)} V_{\left.i^{k}\right)}\right)+ \\
\frac{3 v-1}{4}\left(\frac{2}{\pi \delta^{2} h}\right)^{2} \sum_{i} \frac{\left(\theta_{I}^{\left(i^{k}\right)}-\theta_{I}^{(k)}\right)}{\xi_{\left(i^{k}\right)(k)}^{\left(i^{k}\right.}} n_{I}^{\left(i^{k}\right)(k)} V_{\left(i^{k}\right)} \sum_{i} \frac{\left(\theta_{J}^{\left(i^{k}\right)}-\theta_{J}^{(k)}\right)}{\xi_{\left(i^{k}\right)(k)}} n_{J}^{\left(i^{k}\right)(k)} V_{\left(i^{k}\right)}
\end{array}\right] \\
& +\kappa_{s}^{2} \frac{G}{2} \frac{3}{\pi \delta^{3} h} \times \sum_{i} \frac{\left(w_{\left(i^{k}\right)}-w_{(k)}+\frac{\theta_{I}^{\left(i^{k}\right)}-\theta_{I}^{(k)}}{2} \xi_{\left(i^{k}\right)(k)} n_{I}^{\left(i^{k}\right)(k)}\right)\left(w_{\left(i^{k}\right)}-w_{(k)}+\frac{\theta_{J}^{\left(i^{k}\right)}-\theta_{J}^{(k)}}{2} \xi_{\left(i^{k}\right)(k)} n_{J}^{\left(i^{k}\right)(k)}\right)}{\xi_{\left(i^{k}\right)(k)}} V_{\left(i^{k}\right)}
\end{aligned}
$$




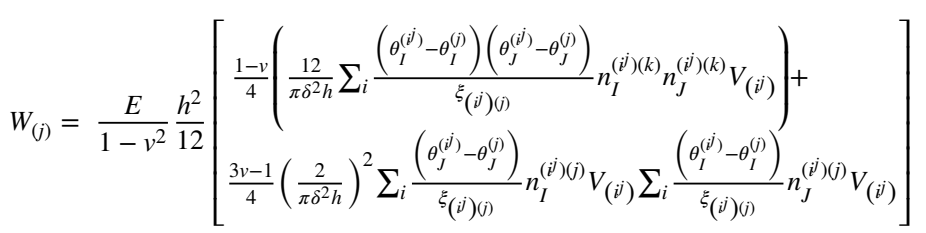

$$
\begin{aligned}
& +\kappa_{s}^{2} \frac{G}{2} \frac{3}{\pi \delta^{3} h} \times \sum_{i} \frac{\left(w_{\left(i^{j}\right)}-w_{(j)}+\frac{\theta_{I}^{\left(j^{j}\right)}-\theta_{I}^{(j)}}{2} \xi_{\left(i^{j}\right)(j)} n_{I}^{\left(i^{j}\right)(j)}\right)\left(w_{\left(i^{j}\right)}-w_{(j)}+\frac{\theta_{J}^{(i j)}-\theta_{J}^{(j)}}{2} \xi_{\left(i^{j}\right)(j)} n_{J}^{\left(i^{j}\right)(j)}\right)}{\xi_{\left(i^{j}\right)(j)}} V_{\left(i^{(j)}\right)}
\end{aligned}
$$

with $n_{1}=\cos \varphi, n_{2}=\sin \varphi$ and $\varphi$ is the orientation of peridynamic bond (interaction).

Inserting Eqs. (36) and (37) into Eq. (35) and renaming the summation indices yield the governing equations of PD Mindlin plate formulation as:

$$
\begin{aligned}
& \rho_{(k)} \frac{h^{2}}{12} \ddot{\theta}_{L}^{(k)}=\frac{E}{1+v} \frac{h^{2}}{12} \frac{12}{\pi \delta^{3} h} \sum_{j} \frac{\left(\theta_{I}^{(j)}-\theta_{I}^{(k)}\right)}{\xi_{(j)(k)}} n_{I}^{(j)(k)} n_{J}^{(j)(k)} V_{\left(i^{k}\right)} \\
& +\left(\frac{E}{1-v^{2}} \frac{3 v-1}{4}\right) \frac{h^{2}}{12}\left(\frac{2}{\pi \delta^{2} h}\right)^{2} \sum_{j} \frac{n_{L}^{(j)(k)}}{\xi_{(j)(k)}}\left(\begin{array}{c}
\frac{\left(\theta_{I}^{\left(i^{k}\right)}-\theta_{I}^{(k)}\right)}{\xi_{\left(i^{k}\right)(k)}} n_{I}^{\left(i^{k}\right)(k)} V_{\left(i^{k}\right)}+ \\
\frac{\left(\theta_{J}^{\left(i^{j}\right)}-\theta_{J}^{(j)}\right)}{\xi_{\left(i^{j}\right)(j)}} n_{J}^{\left(i^{i}\right)(j)} V_{\left(i^{j}\right)}
\end{array}\right) V_{(j)} \\
& -\kappa_{s}^{2} \frac{G}{2} \frac{6}{\pi \delta^{3} h} \sum_{j} \frac{\left(w_{(j)}-w_{(k)}+\frac{\theta_{J}^{(j)}+\theta_{J}^{(k)}}{2} \xi_{(j)(k)} n_{J}^{(j)(k)}\right)}{\xi_{\left(i^{k}\right)(k)}} n_{L}^{(j)(k)} V_{(j)}+b_{\theta_{L}}^{(k)} \\
& \rho_{(k)} \ddot{w}_{(k)}=\kappa_{s}^{2} G \frac{6}{\pi \delta^{3} h} \sum_{j}\left(\frac{w_{(j)}-w_{(k)}}{\xi_{(j)(k)}}+\frac{\theta_{j}^{(k)}+\theta_{j}^{(j)}}{2} n_{j}^{(j)(k)}\right) V_{(j)}+b_{z}^{(k)}
\end{aligned}
$$

In particular, Eqs. (38) and (39) can be simplified for the Poisson's ratio, $v=1 / 3$, as

$$
\begin{aligned}
\rho_{(k)} \frac{h^{2}}{12} \ddot{\theta}_{L}^{(k)}= & c_{b} \sum_{j} \frac{\left(\theta_{I}^{(j)}-\theta_{I}^{(k)}\right)}{\xi_{(j)(k)}} n_{I}^{(j)(k)} n_{L}^{(j)(k)} V_{(j)} \\
& -\frac{c_{s}}{2} \sum_{j}\left(w_{(j)}-w_{(k)}+\frac{\theta_{J}^{(j)}+\theta_{J}^{(k)}}{2} \xi_{(j)(k)} n_{j}^{(j)(k)}\right) n_{L}^{(j)(k)} V_{(j)}+b_{\theta_{L}}^{(k)} \\
\rho_{(k)} \ddot{w}_{(k)}= & c_{s} \sum_{j}\left(\frac{w_{(j)}-w_{(k)}}{\xi_{(j)(k)}}+\frac{\theta_{J}^{(k)}+\theta_{J}^{(j)}}{2} n_{J}^{(j)(k)}\right) V_{(j)}+b_{z}^{(k)}
\end{aligned}
$$

where $c_{b}$ and $c_{s}$ represent PD material parameters related with bending and transverse shear deformations, respectively, which are defined as

$$
c_{b}=\frac{3 E h}{4 \pi \delta^{3}}
$$


and

$$
c_{s}=\frac{9 \kappa_{s}^{2} E}{4 \pi \delta^{3} h}
$$

\section{Boundary Conditions}

As shown in Fig. 3, boundary conditions are imposed by creating a fictitious domain, $R_{c}$, which is located outside of the real domain, $R$. The thickness of this fictitious layer can be specified as twice of the horizon size if $v \neq 1 / 3$, or the size of the horizon if $v=1 / 3$.

\subsection{Clamped Boundary Condition}

The clamped boundary condition can be imposed by specifying zero transverse displacement and zero rotation for the material points at the clamped boundary as

$$
\begin{gathered}
w=0 \\
\theta_{I}=0
\end{gathered}
$$

In this study, these conditions can be obtained via mirror image of the transverse displacements of the material points adjacent to the clamped end and anti-symmetric image of rotation fields as shown in Fig. 4 as

$$
\begin{gathered}
w_{(n)(i)}=w_{(n)\left(i^{*}\right)} \\
w_{(n)(1)}=w_{(n)\left(1^{*}\right)}=0 \\
\theta_{L}^{(n)(i)}=-\theta_{L}^{(n)\left(i^{*}\right)} \quad \text { for }\left\{\begin{array}{cc}
i=1,2, \cdots, 6 & \text { if } v \neq 1 / 3 \\
i=1,2,3 & \text { if } v=1 / 3
\end{array}\right.
\end{gathered}
$$

Fig. 3 Real domain, $R$ and fictitious region, $R_{c}$

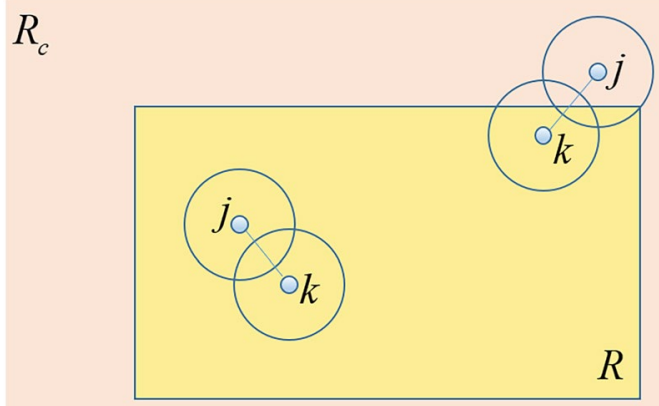




\subsection{Simply Supported Boundary Condition}

The simply supported boundary condition can be obtained by imposing zero transverse displacement and curvature for the material point adjacent to the constrained boundary. The constraint condition related with transverse displacement can be achieved by imposing antisymmetrical transverse displacement fields in the fictitious region as shown in Fig. 5 which can be expressed as

$$
w_{(n)(i)}=-w_{(n)\left(i^{*}\right)} \text { for }\left\{\begin{array}{cc}
i=1,2, \cdots, 6 & \text { if } v \neq 1 / 3 \\
i=1,2,3 & \text { if } v=1 / 3
\end{array}\right.
$$

The curvature condition can be defined as

$$
\theta_{I}^{(n)(i)}=\theta_{I}^{(n)\left(i^{*}\right)} \text { and } \theta_{J}^{(n)(i)}=-\theta_{J}^{(n)\left(i^{*}\right)}(I \neq J) \quad \text { for }\left\{\begin{array}{cc}
i=1,2, \cdots, 6 & \text { if } v \neq 1 / 3 \\
i=1,2,3 & \text { if } v=1 / 3
\end{array}\right.
$$

\section{Numerical Results}

\subsection{Mindlin Plate Subjected to Simply Supported Boundary Conditions}

As the first numerical example, a simply supported Mindlin plate is considered as shown in Fig. 6. The plate has a length and width of $L=W=1 \mathrm{~m}$. The thickness of the plate is specified as $h=0.1 \mathrm{~m}$. The Young's modulus is $E=200 \mathrm{GPa}$ and Poisson's ratio is $v=0.3$. The shear coefficient is used as $\kappa_{s}^{2}=\frac{\pi^{2}}{12}$. For the discretisation of the model, 101 points are used both in directions. The discretisation size is $\Delta x=\frac{1}{101} \mathrm{~m}$. The horizon size is chosen as $\delta=3.606 \Delta x$. A distributed transverse load of $p=100 \mathrm{~N} / \mathrm{m}$ is applied along a row of material points through the central line as a body load of $b_{z}=\frac{p W}{101 \Delta W}=1.01 \times 10^{5} \mathrm{~N} / \mathrm{m}^{3}$ as shown in Fig. 7.

The FE model is generated by using SHELL181 element of ANSYS with $50 \times 50$ elements. As depicted in Figs. 8 and 9, the PD solution of the transverse displacement, $w$, and rotations, $\theta_{L}$, along the central $x$ - and $y$-axes are compared with results from the FE

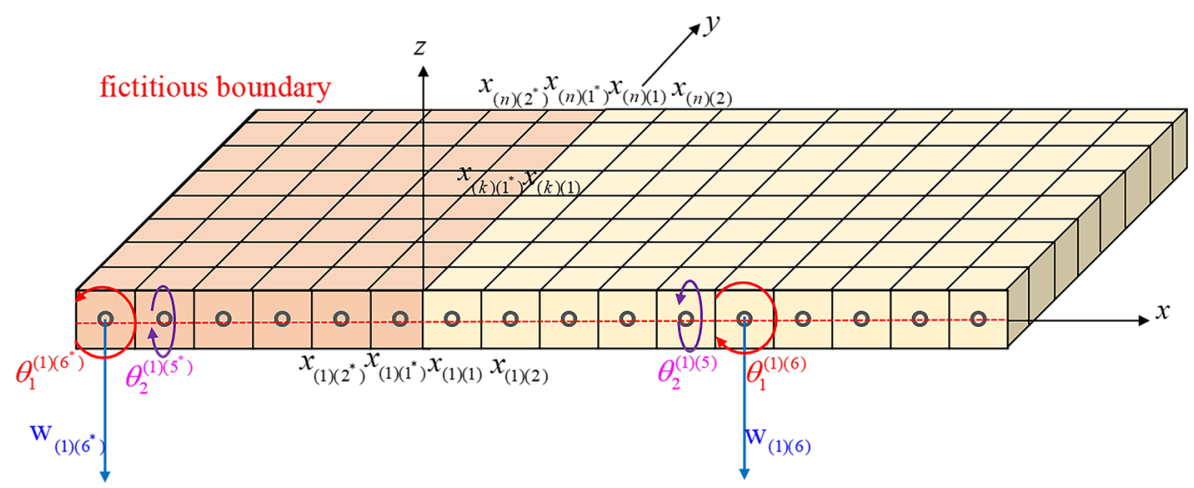

deformed axis

Fig. 4 Application of clamped boundary condition 


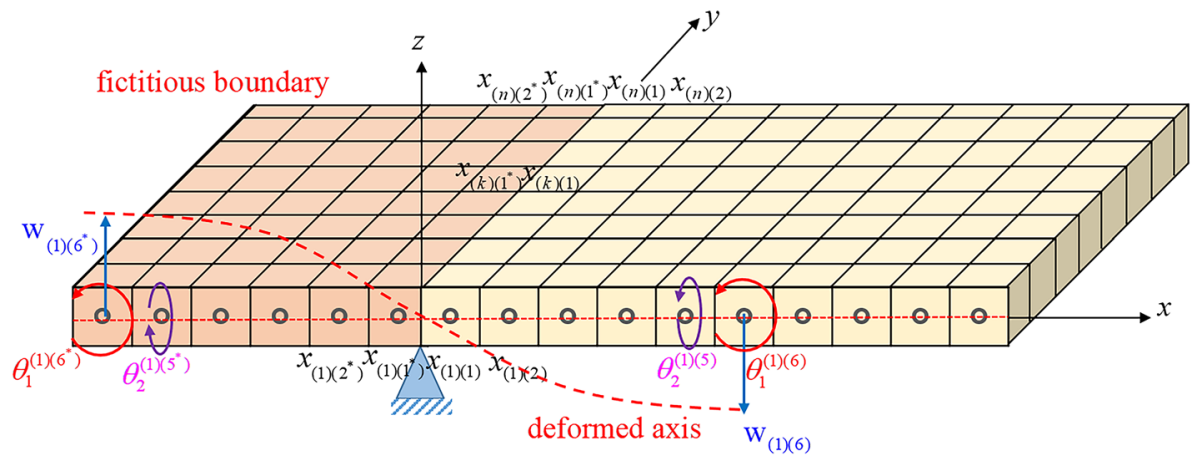

Fig. 5 Application of simply supported boundary condition

method. According to this comparison, it can be concluded that the PD and the FE method results agree well with each other.

\subsection{Mindlin Plate Subjected to Clamped Boundary Conditions}

In the second case, the same problem is considered as in the previous example case except the boundary conditions being clamped instead of simply supported as shown in Fig. 10. As depicted in Figs. 11 and 12, the PD solution of the transverse displacement, $w$, and rotations, $\theta_{L}$, along the central $x$ - and $y$ - axes are compared with the FE method results, and there is a very good agreement between the PD and the FE method results.

\subsection{Mindlin Plate Subjected to Mixed (Clamped-Simply Supported) Boundary Conditions}

In this final numerical case, as opposed to first and second numerical cases, Mindlin plate is subjected to mixed (clamped-simply supported) boundary conditions as shown in Fig. 13, and the PD solution of the transverse displacement $w$, and rotations, $\theta_{L}$, along the central $x$ - and $y$-axes is compared with the FE method results. As depicted in Figs. 14 and 15, the PD and the FE method results agree very well with each other.

Finally, the capability of the current formulation was demonstrated for a different Poisson's ratio of 0.2. As demonstrated in Figs. 16 and 17, the transverse displacement $w$, and rotations, $\theta_{L}$, along the central $x$ - and $y-$ axes results obtained from current formulation compare very well with results obtained from FE analysis. This shows that the current formulation does not have any limitation on material constants.

Fig. 6 Simply supported Mindlin plate and loading condition

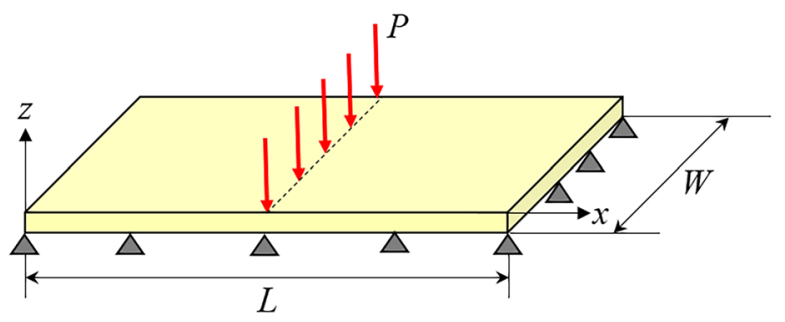




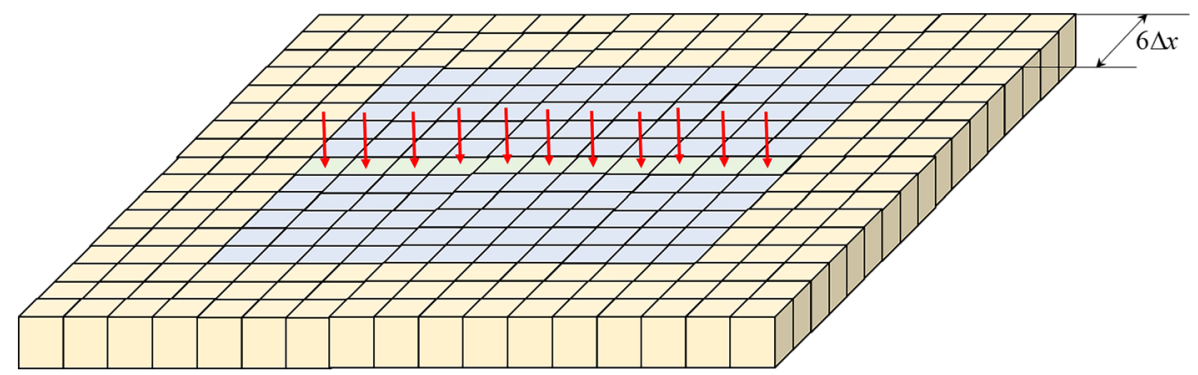

Fig. 7 Discretisation and application of the body load

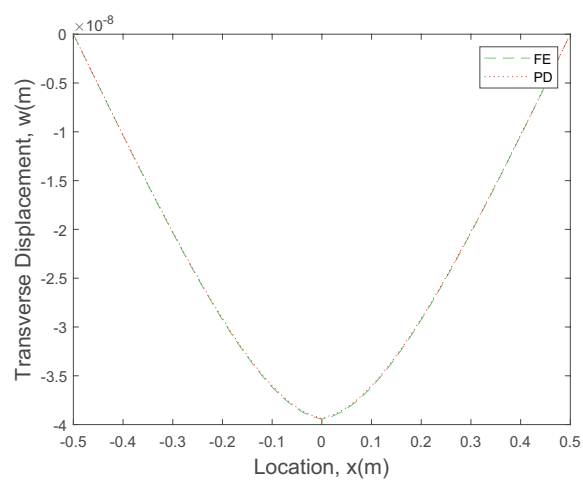

(a)

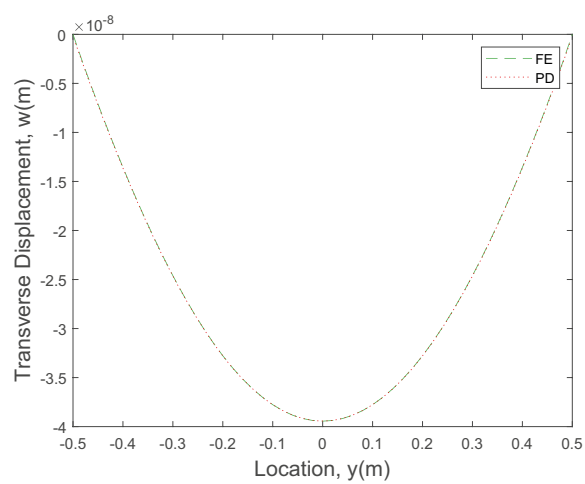

(b)

Fig. 8 Variation of transverse displacements along the central $\mathbf{a} x$-axis, $\mathbf{b} y$-axis

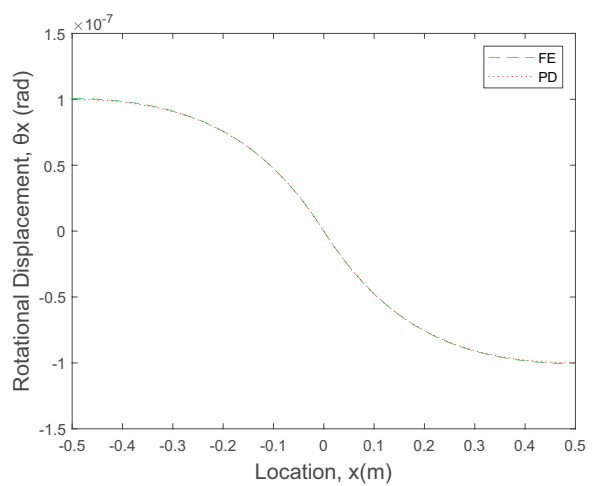

(a)

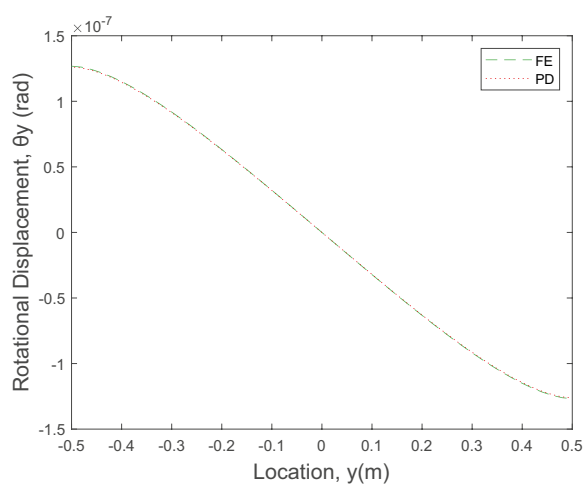

(b)

Fig. 9 Variation of rotations along the central $\mathbf{a} x$ - axis, $\mathbf{b} y$ - axis 
Fig. 10 Clamped Mindlin plate and loading condition

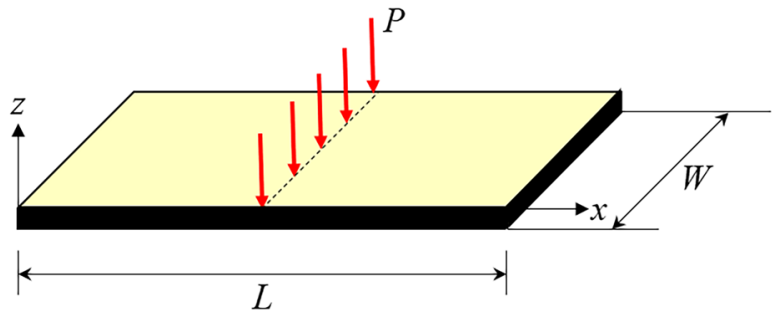

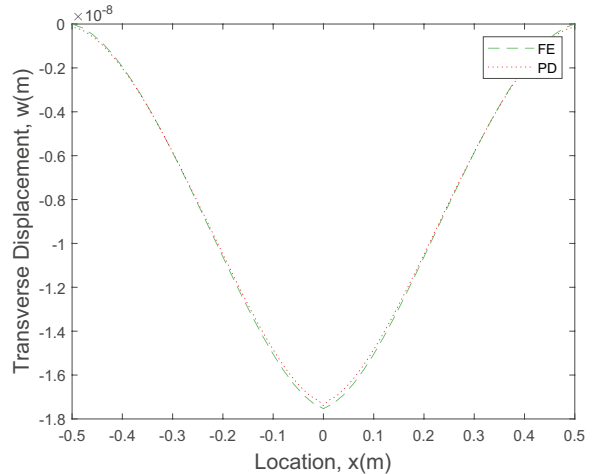

(a)

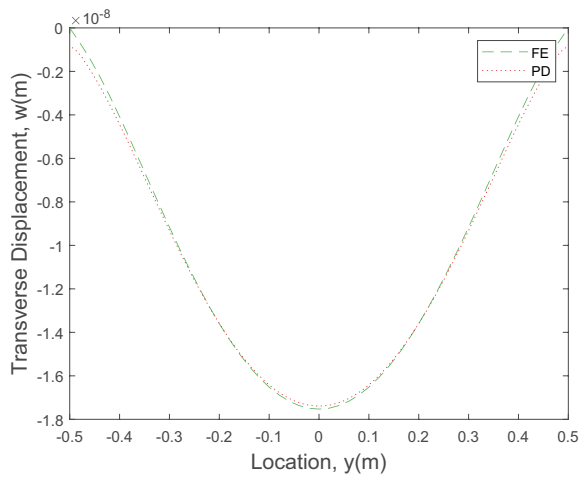

(b)

Fig. 11 Variation of transverse displacements along the central a $x$ - axis, b $y$ - axis

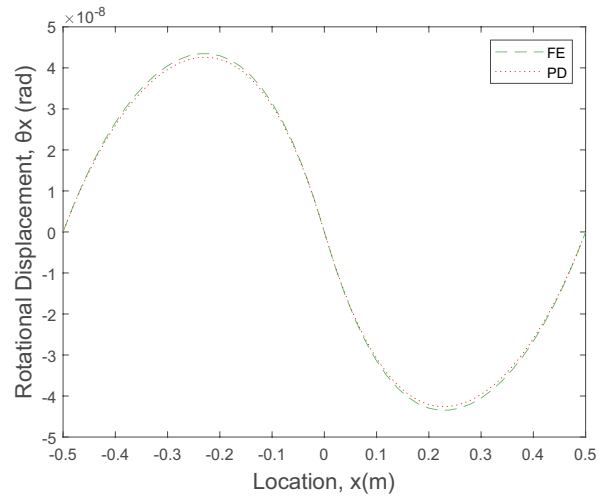

(a)

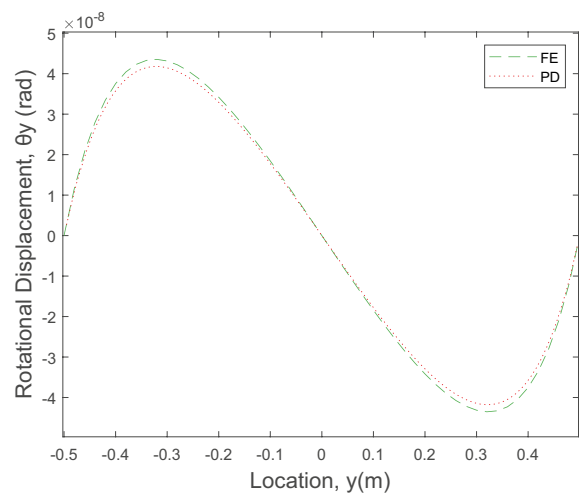

(b)

Fig. 12 Variation of rotations along the central $\mathbf{a} x$-axis, $\mathbf{b}$

Fig. 13 Mindlin plate subjected to mixed (clamped - simply supported) boundary conditions

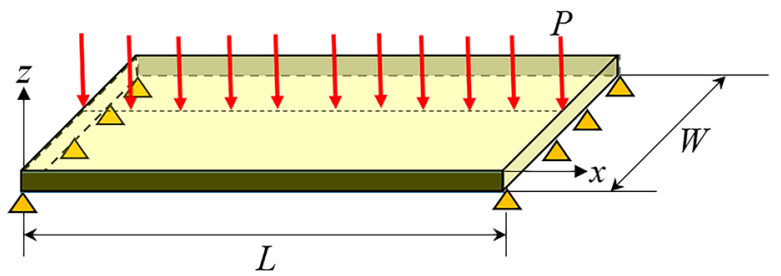




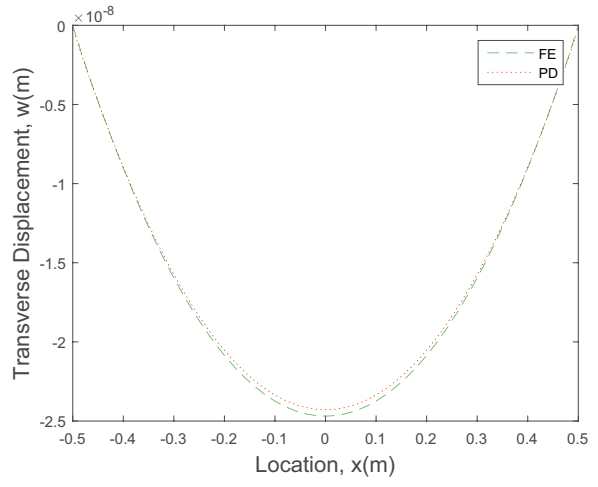

(a)

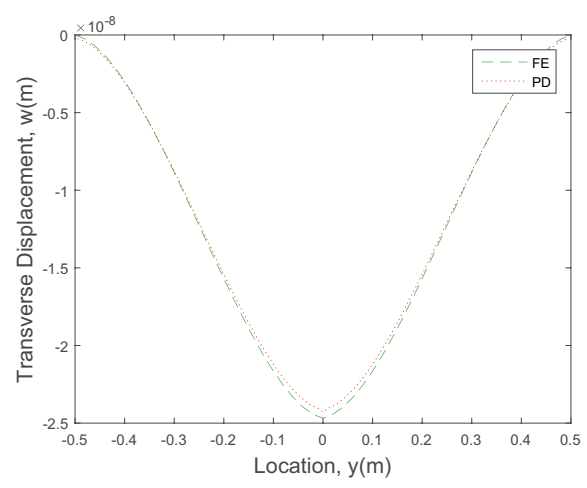

(b)

Fig. 14 Variation of transverse displacements along the central a $x$ - axis, b $y$ - axis

\section{Conclusions}

In this study, a new Mindlin plate formulation was developed. In the current formulation, there is no limitation on material constants as in bond-based peridynamics. Euler-Lagrange equation was utilised in conjunction with Taylor's expansion to determine the equations of motion. Three different numerical examples were considered for a Mindlin plate subjected to different boundary conditions. Peridynamic results were compared against results obtained from finite element analysis, and a good agreement was obtained between the two approaches verifying the newly developed formulation.

\section{Appendix}

As explained above, the strain energy density function of Mindlin plate can be expressed as:

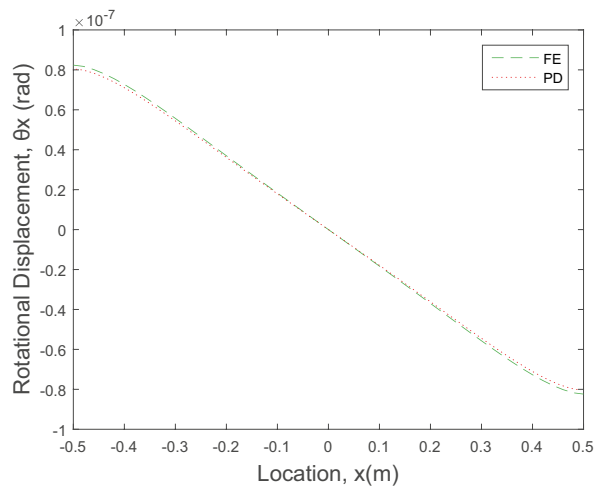

(a)

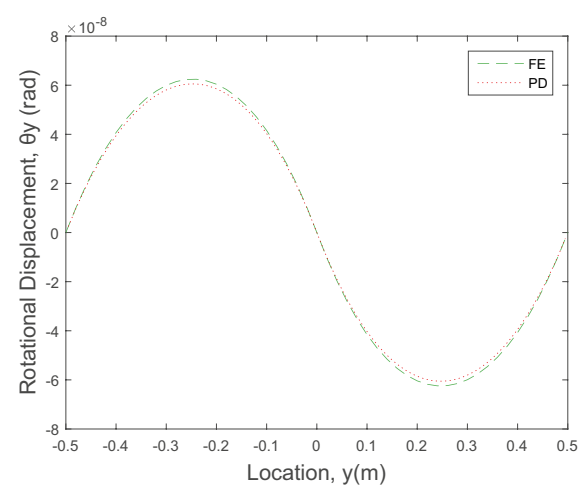

(b)

Fig. 15 Variation of rotations along the central $\mathbf{a} x$ - axis, $\mathbf{b} y$-axis 


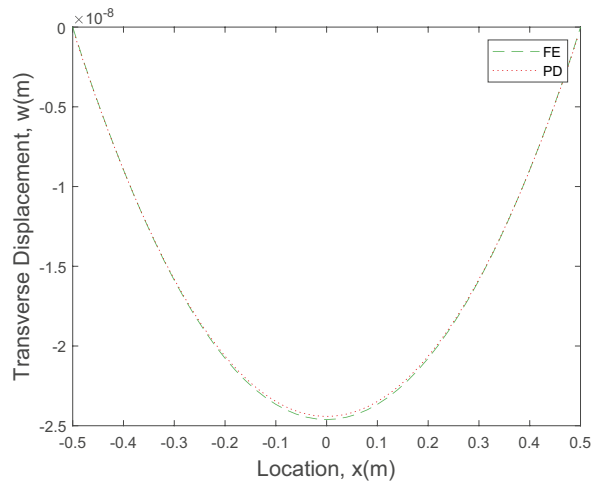

(a)

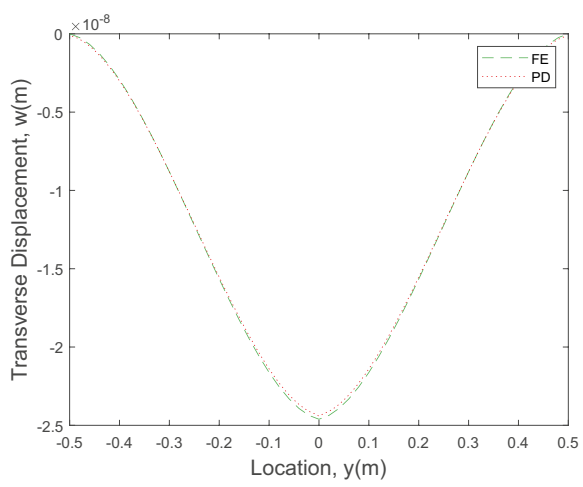

(b)

Fig. 16 Variation of transverse displacements along the central $\mathbf{a} x$ - axis, $\mathbf{b}$

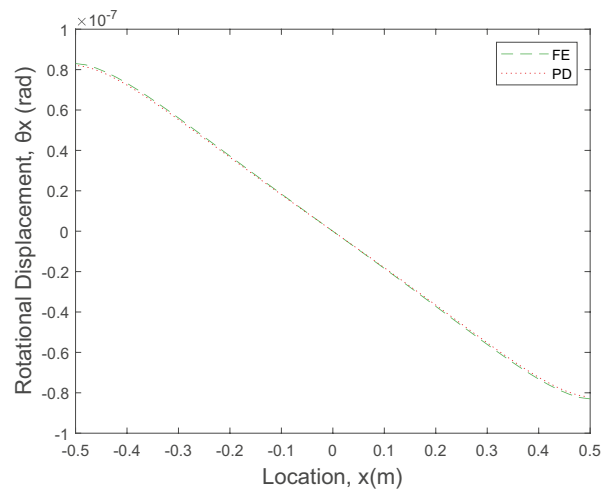

(a)

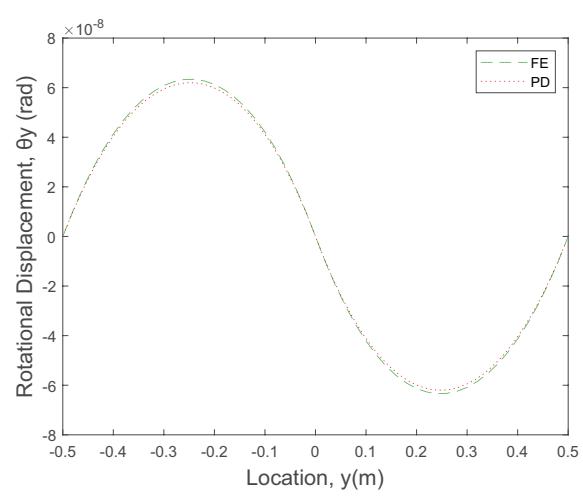

(b)

Fig. 17 Variation of rotations along the central $\mathbf{a}, \mathbf{b} y$ - axis

Fig. 18 Peridynamic interaction between two material points inside the horizon
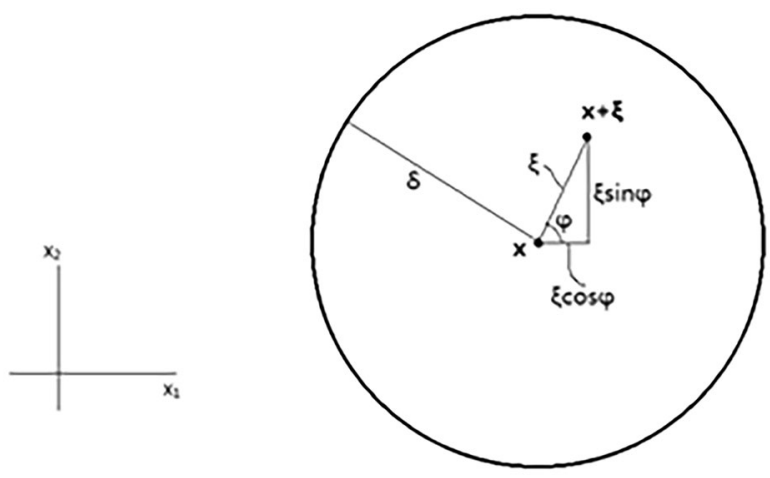


$$
\begin{aligned}
W= & \frac{E}{1-v^{2}} \frac{h^{2}}{12}\left[\frac{1-v}{4}\left(\frac{\partial \theta_{I}}{\partial x_{J}} \frac{\partial \theta_{I}}{\partial x_{J}}+\frac{\partial \theta_{I}}{\partial x_{J}} \frac{\partial \theta_{J}}{\partial x_{I}}+\frac{\partial \theta_{I}}{\partial x_{I}} \frac{\partial \theta_{J}}{\partial x_{J}}\right)+\frac{3 v-1}{4} \frac{\partial \theta_{I}}{\partial x_{I}} \frac{\partial \theta_{J}}{\partial x_{J}}\right] \\
& +\kappa_{s}^{2} \frac{G}{2}\left(\theta_{I}+\frac{\partial w}{\partial x_{I}}\right)\left(\theta_{I}+\frac{\partial w}{\partial x_{I}}\right)
\end{aligned}
$$

In order to obtain the strain energy density function in PD form, it is necessary to transform each differential terms into their equivalent nonlocal form. This can be achieved by utilizing Taylor expansion as:

As shown in Fig. 18, function of rotations, $\theta$, can be Taylor expanded up to 1st-order terms about point $\mathbf{x}$ as:

$$
\begin{gathered}
\theta_{I}(\mathbf{x}+\xi)-\theta_{I}(\mathbf{x})=\frac{\partial \theta_{I}(\mathbf{x})}{\partial x_{J}} \xi n_{J} \\
\theta_{K}(\mathbf{x}+\xi)-\theta_{K}(\mathbf{x})=\frac{\partial \theta_{K}(\mathbf{x})}{\partial x_{L}} \xi n_{L}
\end{gathered}
$$

where $\xi=|\xi|$, and unit direction vector $\mathbf{n}$ is defined as

$$
\mathrm{n}=\left\{\begin{array}{l}
\mathrm{n}_{1} \\
\mathrm{n}_{2}
\end{array}\right\}=\left\{\begin{array}{c}
\cos \varphi \\
\sin \varphi
\end{array}\right\}
$$

with $\varphi$ being the orientation of peridynamic interaction (bond).

Multiplying Eq. (52) with (53) gives

$$
\frac{\left[\theta_{I}(\mathbf{x}+\xi)-\theta_{I}(\mathbf{x})\right]\left[\theta_{K}(\mathbf{x}+\xi)-\theta_{K}(\mathbf{x})\right]}{\xi}=\frac{\partial \theta_{I}(\mathbf{x})}{\partial x_{J}} \frac{\partial \theta_{K}(\mathbf{x})}{\partial x_{L}} \xi n_{J} n_{L}
$$

Multiplying both sides of Eq. (55) twice by directional vector yields

$$
\frac{\left[\theta_{I}(\mathbf{x}+\xi)-\theta_{I}(\mathbf{x})\right]\left[\theta_{K}(\mathbf{x}+\xi)-\theta_{K}(\mathbf{x})\right]}{\xi} n_{R} n_{S}=\frac{\partial \theta_{I}(\mathbf{x})}{\partial x_{J}} \frac{\partial \theta_{K}(\mathbf{x})}{\partial x_{L}} \xi n_{J} n_{L} n_{R} n_{S}
$$

Considering $\mathbf{x}$ as a fixed point, integrating both sides of Eq. (56) over a circular domain with centre of $\mathbf{x}$ and radius of $\delta$ gives:

$$
\begin{aligned}
& \int_{0}^{2 \pi} \int_{0}^{\delta} \frac{\left[\theta_{I}(\mathbf{x}+\xi)-\theta_{I}(\mathbf{x})\right]\left[\theta_{K}(\mathbf{x}+\xi)-\theta_{K}(\mathbf{x})\right]}{\xi} n_{R} n_{S} \xi d \xi d \varphi \\
= & \frac{\partial \theta_{I}(\mathbf{x})}{\partial x_{J}} \frac{\partial \theta_{K}(\mathbf{x})}{\partial x_{L}} \int_{0}^{2 \pi} \int_{0}^{\delta} \xi n_{J} n_{L} n_{R} n_{S} \xi d \xi d \varphi \\
= & \frac{\partial \theta_{I}(\mathbf{x})}{\partial x_{J}} \frac{\partial \theta_{K}(\mathbf{x})}{\partial x_{L}}\left(\delta_{J L} \delta_{R S}+\delta_{J R} \delta_{L S}+\delta_{J S} \delta_{L R}\right) \\
= & \left(\frac{\partial \theta_{I}(\mathbf{x})}{\partial x_{J}} \frac{\partial \theta_{K}(\mathbf{x})}{\partial x_{J}} \delta_{R S}+\frac{\partial \theta_{I}(\mathbf{x})}{\partial x_{R}} \frac{\partial \theta_{K}(\mathbf{x})}{\partial x_{S}}+\frac{\partial \theta_{I}(\mathbf{x})}{\partial x_{S}} \frac{\partial \theta_{K}(\mathbf{x})}{\partial x_{R}}\right)
\end{aligned}
$$

Multiplying both sides of Eq. (57) by $\delta_{R I} \delta_{S K}$ results in: 


$$
\begin{aligned}
& \int_{0}^{2 \pi} \int_{0}^{\delta} \frac{\left[\theta_{I}(\mathbf{x}+\xi)-\theta_{I}(\mathbf{x})\right]\left[\theta_{K}(\mathbf{x}+\xi)-\theta_{K}(\mathbf{x})\right]}{\xi} n_{R} n_{S} \delta_{R I} \delta_{S K} \xi d \xi d \varphi \\
& =\frac{\pi \delta^{3}}{12}\left(\frac{\partial \theta_{I}(\mathbf{x})}{\partial x_{J}} \frac{\partial \theta_{K}(\mathbf{x})}{\partial x_{J}} \delta_{R S}+\frac{\partial \theta_{I}(\mathbf{x})}{\partial x_{R}} \frac{\partial \theta_{K}(\mathbf{x})}{\partial x_{S}}+\frac{\partial \theta_{I}(\mathbf{x})}{\partial x_{S}} \frac{\partial \theta_{K}(\mathbf{x})}{\partial x_{R}}\right) \delta_{R I} \delta_{S K}
\end{aligned}
$$

Rearranging the dummy indices gives:

$$
\begin{aligned}
& \frac{\partial \theta_{I}(\mathbf{x})}{\partial x_{J}} \frac{\partial \theta_{I}(\mathbf{x})}{\partial x_{J}}+\frac{\partial \theta_{I}(\mathbf{x})}{\partial x_{I}} \frac{\partial \theta_{J}(\mathbf{x})}{\partial x_{J}}+\frac{\partial \theta_{I}(\mathbf{x})}{\partial x_{J}} \frac{\partial \theta_{J}(\mathbf{x})}{\partial x_{I}} \\
& =\frac{12}{\pi \delta^{3}} \int_{0}^{2 \pi} \int_{0}^{\delta} \frac{\left[\theta_{I}(\mathbf{x}+\xi)-\theta_{I}(\mathbf{x})\right]\left[\theta_{J}(\mathbf{x}+\xi)-\theta_{J}(\mathbf{x})\right]}{\xi} n_{I} n_{J} \xi d \xi d \varphi
\end{aligned}
$$

which can be written in the discretised form as

$$
\frac{\partial \theta_{I}^{(k)}}{\partial x_{J}} \frac{\partial \theta_{I}^{(k)}}{\partial x_{J}}+\frac{\partial \theta_{I}^{(k)}}{\partial x_{I}} \frac{\partial \theta_{J}^{(k)}}{\partial x_{J}}+\frac{\partial \theta_{I}^{(k)}}{\partial x_{J}} \frac{\partial \theta_{J}^{(k)}}{\partial x_{I}}=\frac{12}{\pi \delta^{3} h} \sum_{\mathrm{i}} \frac{\left[\theta_{I}^{\left(i^{k}\right)}-\theta_{I}^{(k)}\right]\left[\theta_{J}^{\left(i^{k}\right)}-\theta_{J}^{(k)}\right]}{\xi_{\left(i^{k}\right)(k)}} n_{I}^{\left(i^{k}\right)(k)} n_{J}^{\left(i^{k}\right)(k)} V_{\left(i^{k}\right)}
$$

where $h$ represents the thickness of the beam in this study.

Recalling Eq. (52):

$$
\theta_{I}(\mathbf{x}+\xi)-\theta_{I}(\mathbf{x})=\frac{\partial \theta_{I}(\mathbf{x})}{\partial x_{J}} \xi n_{J}
$$

and multiplying Eq. (61) by a directional vector gives:

$$
\frac{\theta_{I}(\mathbf{x}+\xi)-\theta_{I}(\mathbf{x})}{\xi} n_{K}=\frac{\partial \theta_{I}(\mathbf{x})}{\partial x_{J}} n_{J} n_{K}
$$

Considering $\mathbf{x}$ as a fixed point, integrating both sides of Eq. (62) over a circular domain with centre of $\mathbf{x}$ and radius of $\delta$ yields:

$$
\int_{0}^{2 \pi} \int_{0}^{\delta} \frac{\theta_{I}(\mathbf{x}+\xi)-\theta_{I}(\mathbf{x})}{\xi} n_{K} \xi d \xi d \varphi=\frac{\partial \theta_{I}(\mathbf{x})}{\partial x_{J}} \int_{0}^{2 \pi} \int_{0}^{\delta} n_{J} n_{K} \xi d \xi d \varphi=\frac{\partial \theta_{I}(\mathbf{x})}{\partial x_{J}} \frac{\pi \delta^{2}}{2} \delta_{J K}=\frac{\partial \theta_{I}(\mathbf{x})}{\partial x_{K}} \frac{\pi \xi^{2}}{2}
$$

which can be rewritten as

$$
\frac{\partial \theta_{I}(\mathbf{x})}{\partial x_{K}}=\frac{2}{\pi \delta^{2}} \int_{0}^{2 \pi} \int_{0}^{\delta} \frac{\theta_{I}(\mathbf{x}+\xi)-\theta_{I}(\mathbf{x})}{\xi} n_{K} \xi d \xi d \varphi
$$

Multiplying both sides of Eq. (64) by $\delta_{I K}$ gives

$$
\frac{\partial \theta_{I}(\mathbf{x})}{\partial x_{I}}=\frac{2}{\pi \delta^{2}} \int_{0}^{2 \pi} \int_{0}^{\delta} \frac{\theta_{I}(\mathbf{x}+\xi)-\theta_{I}(\mathbf{x})}{\xi} n_{I} \xi d \xi d \varphi
$$

Rewriting Eq. (65) with a different index gives: 


$$
\frac{\partial \theta_{J}(\mathbf{x})}{\partial x_{J}}=\frac{2}{\pi \delta^{2}} \int_{0}^{2 \pi} \int_{0}^{\delta} \frac{\theta_{J}(\mathbf{x}+\xi)-\theta_{J}(\mathbf{x})}{\xi} n_{J} \xi d \xi d \varphi
$$

Multiplying Eq. (65) with (66) yields:

$$
\frac{\partial \theta_{I}(\mathbf{x})}{\partial x_{I}} \frac{\partial \theta_{J}(\mathbf{x})}{\partial x_{J}}=\left(\frac{2}{\pi \delta^{2}}\right)^{2} \int_{0}^{2 \pi} \int_{0}^{\delta} \frac{\theta_{I}(\mathbf{x}+\xi)-\theta_{I}(\mathbf{x})}{\xi} n_{I} \xi d \xi d \varphi \int_{0}^{2 \pi} \int_{0}^{\delta} \frac{\theta_{J}(\mathbf{x}+\xi)-\theta_{J}(\mathbf{x})}{\xi} n_{J} \xi d \xi d \varphi
$$

which can be written in discretised form as

$$
\frac{\partial \theta_{i}^{(k)}}{\partial x_{I}} \frac{\partial \theta_{J}^{(k)}}{\partial x_{J}}=\left(\frac{2}{\pi \delta^{2}}\right)^{2} \sum_{i} \frac{\theta_{I}^{\left(i^{k}\right)}-\theta_{I}^{(k)}}{\xi_{\left(i^{k}\right)(k)}} n_{I}^{\left(i^{k}\right)(k)} V_{\left(i^{k}\right)} \sum_{i} \frac{\theta_{J}^{\left(i^{k}\right)}-\theta_{J}^{(k)}}{\xi_{\left(i^{k}\right)(k)}} n_{I}^{\left(i^{k}\right)(k)} V_{\left(i^{k}\right)}
$$

Similar to Eq. (52), the following relationship can be established as:

$$
w(\mathbf{x}+\xi)-w(\mathbf{x})=\frac{\partial w(\mathbf{x})}{\partial x_{J}} \xi n_{J}
$$

The rotations of material point $\mathbf{x}$ can be estimated as taking the average rotation of point $\mathbf{x}$ and its family member point $\mathbf{x}+\boldsymbol{\xi}$ as:

$$
\frac{\theta_{I}(\mathbf{x}+\xi)+\theta_{I}(\mathbf{x})}{2}=\theta_{I}(\mathbf{x})
$$

If Eq. (70) is multiplied by $\xi n_{I}$ :

$$
\frac{\theta_{I}(\mathbf{x}+\xi)-\theta_{I}(\mathbf{x})}{2} \xi n_{I}=\theta_{I}(\mathbf{x}) \xi n_{I}
$$

and added with (69) yields:

$$
w(\mathbf{x}+\xi)-w(\mathbf{x})+\frac{\theta_{I}(\mathbf{x}+\xi)+\theta_{I}(\mathbf{x})}{2} \xi n_{I}=\frac{\partial w(\mathbf{x})}{\partial x_{J}} \xi n_{J}+\theta_{I}(\mathbf{x}) \xi n_{I}
$$

Rewriting Eq. (72) with a different index results in:

$$
w(\mathbf{x}+\xi)-w(\mathbf{x})+\frac{\theta_{J}(\mathbf{x}+\xi)+\theta_{J}(\mathbf{x})}{2} \xi n_{J}=\frac{\partial w(\mathbf{x})}{\partial x_{J}}=\xi n_{J}+\theta_{J}(\mathbf{x}) \xi n_{J}
$$

Multiplying Eq. (72) with (73) and then dividing each term by $\xi$ yields:

$$
\begin{aligned}
& \frac{\left[w(\mathbf{x}+\xi)-w(\mathbf{x})+\frac{\theta_{J}(\mathbf{x}+\xi)+\theta_{J}(\mathbf{x})}{2} \xi n_{J}\right]\left[w(\mathbf{x}+\xi)-w(\mathbf{x})+\frac{\theta_{I}(\mathbf{x}+\xi)+\theta_{I}(\mathbf{x})}{2} \xi n_{I}\right]}{\xi} \\
& =\left(\frac{\partial w(\mathbf{x})}{\partial x_{I}}+\theta_{I}(\mathbf{x})\right)\left(\frac{\partial w(\mathbf{x})}{\partial x_{J}}+\theta_{J}(\mathbf{x})\right) n_{I} n_{J}
\end{aligned}
$$

Considering $\mathbf{x}$ as a fixed point, integrating both sides of Eq. (74) over a circular domain with centre of $\mathbf{x}$ and radius of $\delta$ results in 


$$
\begin{aligned}
& \int_{0}^{2 \pi} \int_{0}^{\delta} \frac{\left[w(\mathbf{x}+\xi)-w(\mathbf{x})+\frac{\theta_{J}(\mathbf{x}+\xi)+\theta_{J}(\mathbf{x})}{2} \xi n_{J}\right]\left[w(\mathbf{x}+\xi)-w(\mathbf{x})+\frac{\theta_{I}(\mathbf{x}+\xi)+\theta_{I}(\mathbf{x})}{2} \xi n_{I}\right]}{\xi} \xi d \xi d \varphi \\
& =\left(\frac{\partial w(\mathbf{x})}{\partial x_{I}}+\theta_{I}(\mathbf{x})\right)\left(\frac{\partial w(\mathbf{x})}{\partial x_{J}}+\theta_{J}(\mathbf{x})\right) \int_{0}^{2 \pi} \int_{0}^{\delta} n_{I} n_{J} \xi d \xi d \varphi \\
& =\left(\frac{\partial w(\mathbf{x})}{\partial x_{I}}+\theta_{I}(\mathbf{x})\right)\left(\frac{\partial w(\mathbf{x})}{\partial x_{J}}+\theta_{J}(\mathbf{x})\right) \frac{\pi \delta^{3}}{3} \delta_{I J}=\frac{\pi \delta^{2}}{3}\left(\frac{\partial w(\mathbf{x})}{\partial x_{I}}+\theta_{I}(\mathbf{x})\right)^{2}
\end{aligned}
$$

which gives

$$
\begin{aligned}
& \left(\frac{\partial \mathrm{w}(\mathbf{x})}{\partial x_{I}}+\theta_{I}(\mathbf{x})\right)^{2} \\
& =\frac{3}{\pi \delta^{2}} \int_{0}^{2 \pi} \int_{0}^{\delta} \frac{\left[w(\mathbf{x}+\xi)-w(\mathbf{x})+\frac{\theta_{J}(\mathbf{x}+\xi)+\theta_{J}(\mathbf{x})}{2} \xi n_{J}\right]\left[w(\mathbf{x}+\xi)-w(\mathbf{x})+\frac{\theta_{I}(\mathbf{x}+\xi)+\theta_{I}(\mathbf{x})}{2} \xi n_{I}\right]}{\xi} \xi d \xi d \varphi
\end{aligned}
$$

Equation (76) can be written in discretised form as

$$
\begin{aligned}
& w(\mathbf{x}+\xi)-w(\mathbf{x})=\frac{\partial w(\mathbf{x})}{\partial x_{J}} \xi n_{J}\left(\frac{\partial w_{(k)}}{\partial x_{I}}+\theta_{I}^{(k)}\right)^{2} \\
& =\frac{3}{\pi \delta^{3} h} \sum_{i} \frac{\left[w_{\left(i^{k}\right)}-w_{(k)}+\frac{\theta_{I}^{(k)}+\theta_{I}^{\left(i^{k}\right)}}{2} \xi_{\left(i^{k}\right)(k)} n_{I}^{\left(i^{k}\right)(k)}\right]\left[w_{\left(i^{k}\right)}-w_{(k)}+\frac{\theta_{J}^{(k)}+\theta_{J}^{\left(i^{k}\right)}}{2} \xi_{\left(i^{k}\right)(k)} n_{J}^{\left(i^{k}\right)(k)}\right]}{\xi_{\left(i^{k}\right)(k)}}
\end{aligned}
$$

Finally, combining Eqs. (60), (68), (77) and (51) gives the strain energy density of the material point $k$ in PD form as

$$
\begin{aligned}
& W_{(k)}=\frac{E}{1-v^{2}} \frac{h^{2}}{12}\left[\begin{array}{c}
\frac{1-v}{4}\left(\frac{12}{\pi \delta^{3} h} \sum_{i} \frac{\left(\theta_{I}^{\left(i^{k}\right)}-\theta_{I}^{(k)}\right)\left(\theta_{J}^{\left(i^{k}\right)}-\theta_{J}^{(k)}\right)}{\xi_{\left(i^{k}\right)(k)}} n_{I}^{\left(i^{k}\right)(k)} n_{J}^{\left(i^{k}\right)(k)} V_{\left(i^{k}\right)}\right)+ \\
\frac{3 v-1}{4}\left(\frac{2}{\pi \delta^{2} h}\right)^{2} \sum_{i} \frac{\left(\theta_{I}^{\left(i^{k}\right)}-\theta_{I}^{(k)}\right)}{\xi_{\left(i^{k}\right)(k)}} n_{I}^{\left(i^{k}\right)(k)} V_{\left(i^{k}\right)} \sum_{i} \frac{\left(\theta_{J}^{\left(i^{k}\right)}-\theta_{J}^{(k)}\right)}{\xi_{(i k)}(k)} n_{J}^{\left(i^{k}\right)(k)} V_{\left(i^{k}\right)}
\end{array}\right] \\
& +\kappa_{s}^{2} \frac{G}{2} \frac{3}{\pi \delta^{3} h} \times \sum_{i} \frac{\left(w_{\left(i^{k}\right)}-w_{(k)}+\frac{\theta_{I}^{\left(i^{k}\right)}+\theta_{I}^{(k)}}{2} \xi_{\left(i^{k}\right)(k)} n_{I}^{\left(i^{k}\right)(k)}\right)\left(w_{\left(i^{k}\right)}-w_{(k)}+\frac{\theta_{J}^{\left(i^{k}\right)}+\theta_{J}^{(k)}}{2} \xi_{\left(i^{k}\right)(k)} n_{J}^{\left(i^{k}\right)(k)}\right)}{\xi_{\left(i^{k}\right)(k)}} V_{\left(i^{k}\right)}
\end{aligned}
$$

Regarding the strain energy density for the material point $j$, a similar form will hold if we replace the index $k$ with $j$ as: 


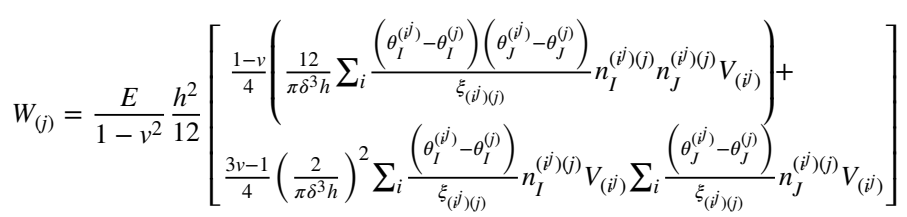

$$
\begin{aligned}
& +\kappa_{s}^{2} \frac{G}{2} \frac{3}{\pi \delta^{3} h} \times \sum_{i} \frac{\left(w_{\left(i^{j}\right)}-w_{(j)}+\frac{\theta_{I}^{\left(i^{j}\right)}+\theta_{I}^{(j)}}{2} \xi_{(i j)(j)} n_{I}^{\left(i^{j}\right)(j)}\right)\left(w_{\left(i^{j}\right)}-w_{(j)}+\frac{\theta_{J}^{\left(i^{j}\right)}+\theta_{J}^{(j)}}{2} \xi_{(i j)(j)} n_{J}^{\left(i^{j}\right)(j)}\right)}{\xi_{\left(i^{j}\right)(j)}} V_{\left(i^{j}\right)}
\end{aligned}
$$

\section{Declarations}

Conflict of Interest The authors declare that they have no conflict of interest.

Open Access This article is licensed under a Creative Commons Attribution 4.0 International License, which permits use, sharing, adaptation, distribution and reproduction in any medium or format, as long as you give appropriate credit to the original author(s) and the source, provide a link to the Creative Commons licence, and indicate if changes were made. The images or other third party material in this article are included in the article's Creative Commons licence, unless indicated otherwise in a credit line to the material. If material is not included in the article's Creative Commons licence and your intended use is not permitted by statutory regulation or exceeds the permitted use, you will need to obtain permission directly from the copyright holder. To view a copy of this licence, visit http://creativecommons.org/licenses/by/4.0/.

\section{References}

1. Silling SA (2000) Reformulation of elasticity theory for discontinuities and long-range forces. J Mech Phys Solids 48(1):175-209

2. Ren H, Zhuang X, Cai Y, Rabczuk T (2016) Dual-horizon peridynamics. Int J Numer Meth Eng 108(12):1451-1476

3. Ren H, Zhuang X, Rabczuk T (2017) Dual-horizon peridynamics: a stable solution to varying horizons. Comput Methods Appl Mech Eng 318:762-782

4. Amani J, Oterkus E, Areias P, Zi G, Nguyen-Thoi T, Rabczuk T (2016) A non-ordinary state-based peridynamics formulation for thermoplastic fracture. Int J Impact Eng 87:83-94

5. Javili A, McBride AT, Steinmann P (2019) Continuum-kinematics-inspired peridynamics. Mechanical problems. J Mech Phys Solids 131:125-146

6. Wang X, Kulkarni SS, Tabarraei A (2019) Concurrent coupling of peridynamics and classical elasticity for elastodynamic problems. Comput Methods Appl Mech Eng 344:251-275

7. Ni T,Zaccariotto M, Zhu QZ, Galvanetto U (2019) Static solution of crack propagation problems in peridynamics. Comput Methods Appl Mech Eng 346:126-151

8. Chowdhury SR, Roy P, Roy D, Reddy JN (2019) A modified peridynamics correspondence principle: removal of zero-energy deformation and other implications. Comput Methods Appl Mech Eng 346:530-549

9. Liu S, Fang G, Liang J, Fu M, Wang B (2020) A new type of peridynamics: element-based peridynamics. Comput Methods Appl Mech Eng 366:113098

10. Diehl P, Prudhomme S, Lévesque M (2019) A review of benchmark experiments for the validation of peridynamics models. Journal of Peridynamics and Nonlocal Modeling 1(1):14-35

11. Katiyar A, Agrawal S, Ouchi H, Seleson P, Foster JT, Sharma MM (2020) A general peridynamics model for multiphase transport of non-Newtonian compressible fluids in porous media. J Comput Phys 402:109075

12. Ozdemir M, Kefal A, Imachi M, Tanaka S, Oterkus E (2020) Dynamic fracture analysis of functionally graded materials using ordinary state-based peridynamics. Compos Struct, p.112296

13. Liu S, Fang G, Liang J, Lv D (2020) A coupling model of XFEM/peridynamics for 2D dynamic crack propagation and branching problems. Theor Appl Fract Mech, p.102573 
14. Mehrmashhadi J, Wang L, Bobaru F (2019) Uncovering the dynamic fracture behavior of PMMA with peridynamics: the importance of softening at the crack tip. Eng Fract Mech 219:106617

15. Shou Y, Zhou X (2020) A coupled thermomechanical nonordinary state-based peridynamics for thermally induced cracking of rocks. Fatigue Fract Eng Mater Struct 43(2):371-386

16. Madenci E, Dorduncu M, Gu X (2019) Peridynamic least squares minimization. Comput Methods Appl Mech Eng 348:846-874

17. Dorduncu M (2019) Stress analysis of laminated composite beams using refined zigzag theory and peridynamic differential operator. Compos Struct 218:193-203

18. Oterkus E, Madenci E (2012) Peridynamics for failure prediction in composites. In 53rd AIAA/ASME/ ASCE/AHS/ASC Structures, Structural Dynamics and Materials Conference 20th AIAA/ASME/AHS Adaptive Structures Conference 14th AIAA, p. 1692

19. Oterkus E, Guven I, Madenci E (2012) Impact damage assessment by using peridynamic theory. Open Engineering 2(4):523-531

20. Wang H, Oterkus E, Oterkus S (2018) Predicting fracture evolution during lithiation process using peridynamics. Eng Fract Mech 192:176-191

21. De Meo D, Oterkus E (2017) Finite element implementation of a peridynamic pitting corrosion damage model. Ocean Eng 135:76-83

22. Gao Y, Oterkus S (2019) Ordinary state-based peridynamic modelling for fully coupled thermoelastic problems. Contin Mech Thermodyn 31(4):907-937

23. Imachi M, Tanaka S, Bui TQ, Oterkus S, Oterkus E (2019) A computational approach based on ordinary state-based peridynamics with new transition bond for dynamic fracture analysis. Eng Fract Mech 206:359-374

24. Diyaroglu C, Oterkus S, Oterkus E, Madenci E (2017) Peridynamic modeling of diffusion by using finiteelement analysis. IEEE Trans Compon Packaging Manuf Technol 7(11):1823-1831

25. Javili A, Morasata R, Oterkus E, Oterkus S (2019) Peridynamics review. Math Mech Solids 24(11):3714-3739

26. Taylor M, Steigmann DJ (2015) A two-dimensional peridynamic model for thin plates. Math Mech Solids 20(8):998-1010

27. O'Grady J, Foster J (2014a) Peridynamic beams: a non-ordinary, state-based model. Int J Solids Struct 51(18):3177-3183

28. O'Grady J, Foster J (2014b) Peridynamic plates and flat shells: a non-ordinary, state-based model. Int J Solids Struct 51(25-26):4572-4579

29. Diyaroglu C, Oterkus E, Oterkus S (2019) An Euler-Bernoulli beam formulation in an ordinary state-based peridynamic framework. Math Mech Solids 24(2):361-376

30. Yang Z, Vazic B, Diyaroglu C, Oterkus E, Oterkus S (2020) A Kirchhoff plate formulation in a state-based peridynamic framework. Math Mech Solids 25(3):727-738

31. Diyaroglu C, Oterkus E, Oterkus S, Madenci E (2015) Peridynamics for bending of beams and plates with transverse shear deformation. Int J Solids Struct 69:152-168

32. Madenci E, Oterkus E (2014) Peridynamic theory and its applications, vol 17. Springer, New York 